\title{
THE BEHAVIOUR OF SQUARE FUNCTIONS FROM ERGODIC THEORY IN $L^{\infty}$
}

\author{
GUIXIANG HONG \\ (Communicated by Alexander Iosevich)
}

\begin{abstract}
In this paper, we analyze carefully the behaviour in $L^{\infty}(\mathbb{R})$ of the square functions $S$ and $S_{\mathcal{I}}$, originating from ergodic theory. First, we show that we can find some function $f \in L^{\infty}(\mathbb{R})$, such that $S f$ equals infinity on a nonzero measurable set. Second, we can find compact supported function $f \in L^{\infty}(\mathbb{R})$ and $\mathcal{I}$ such that $S_{\mathcal{I}} f$ does not belong to $B M O$ space. Finally, we show that $S$ is bounded from $L_{c}^{\infty}$, the space of compactly supported $L^{\infty}(\mathbb{R})$ functions, to $B M O$ space. As a consequence, we solve an open question posed by Jones, Kaufman, Rosenblatt and Wierdl (2000). That is, $S_{\mathcal{I}}$ are uniformly bounded in $L^{p}(\mathbb{R})$ with respect to $\mathcal{I}$ for $2<p<\infty$.
\end{abstract}

\section{INTRODUCTION}

A variety of square functions were introduced in 22 by Jones et al. as tools to deal with variational inequalities, whence measure the speed of the convergence of a sequence of differential averages. To present the square functions in which we are interested in this paper, we need some notation. Let $\sigma_{k}$ be the $k$-th dyadic $\sigma$ algebra in $\mathbb{R}$. That is, $\sigma_{k}$ is generated by the dyadic intervals with side-length equal to $2^{k}$. Denote by $\mathcal{E}_{k}$ the expectation with respect to $\sigma_{k}$. For $x \in \mathbb{R}$, let $I_{k}(x)$

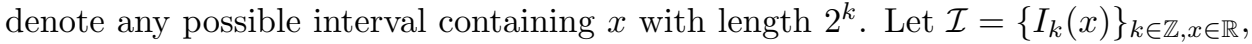
for any finite compact supported function $f$ on $\mathbb{R}$, and define

$$
S_{\mathcal{I}} f(x)=\left(\sum_{k \in \mathbb{Z}}\left|M_{I_{k}(x)} f(x)-\mathcal{E}_{k} f(x)\right|^{2}\right)^{1 / 2},
$$

where

$$
M_{I_{k}(x)} f(x)=2^{-k} \int_{I_{k}(x)} f(y) d y .
$$

In Theorem 2.2 of [2], the authors proved that $S_{\mathcal{I}}$ is bounded in $L^{2}(\mathbb{R})$ uniformly with respect to $\mathcal{I}$. That is, there exists a constant $C>0$ independent of $\mathcal{I}$ such that

$$
\left\|S_{\mathcal{I}} f\right\|_{2} \leq C\|f\|_{2}, \forall f \in L^{2}(\mathbb{R}) .
$$

But in Remark 4.5 of the same paper, the authors observed that for some $\mathcal{I}, S_{\mathcal{I}}$ may not map $L^{\infty}$ to $B M O_{d}$ (the dyadic BMO space on the torus). Hence the interpolation argument cannot be applied, and they leave it as an open question

Received by the editors April 28, 2014 and, in revised form, July 29, 2014.

2010 Mathematics Subject Classification. Primary 42B25; Secondary 47G10.

Key words and phrases. Square function, behaviour in $L^{\infty}$.

The author was supported by MINECO: ICMAT Severo Ochoa project SEV-2011-0087 and ERC Grant StG-256997-CZOSQP (EU). 
(Question 4.7 in the same paper) of whether $S_{\mathcal{I}}$ is bounded in $L^{p}(\mathbb{R})$ uniformly with respect to $\mathcal{I}$ for $2<p<\infty$.

In this paper, we give a positive answer to this question. In order to present our approach, we need more notation. Let $\mathcal{I}_{k}$ be the set of intervals containing the origin with length $2^{k}$. We will consider the following square function:

$$
S f(x)=\left(\sum_{k \in \mathbb{Z}} \sup _{I \in \mathcal{I}_{k}}\left|M_{I+x} f(x)-\mathcal{E}_{k} f(x)\right|^{2}\right)^{1 / 2} .
$$

It is clear that for all $\mathcal{I}, S_{\mathcal{I}} f(x) \leq S f(x)$ for all $f$ and almost every $x \in \mathbb{R}$. Hence for all $1<p<\infty, L^{p}$-boundedness of $S$ implies the uniform $L^{p}$-boundedness of $S_{\mathcal{I}}$, since the spaces $L^{p}$ are Köthe function spaces. Hence it suffices to prove $S$ is bounded on $L^{p}(\mathbb{R})$ for all $2<p<\infty$.

It is known from Theorem A' of $\left[3\right.$ that $S$ is bounded on $L^{2}(\mathbb{R})$, i.e.,

$$
\|S f\|_{2} \leq C\|f\|_{2}, \forall f \in L^{2}(\mathbb{R}),
$$

for some positive constant $C$. Hence the $L^{p}$-boundedness would be obtained by interpolation, if we could show the $\left(L^{\infty}, B M O_{d}\right)$-boundedness of $S$. However, as recalled previously, for some $\mathcal{I}, S_{\mathcal{I}}$ may not be bounded from $L^{\infty}$ to $B M O_{d}$. Now a key observation is that the $\left(L^{\infty}, B M O_{d}\right)$-boundedness of $S$ may still survive, since $B M O$ spaces are not Köthe function spaces.

Therefore, in Section 2, we carefully analyze the behaviour of $S_{\mathcal{I}}$ and $S$ in $L^{\infty}$. The results we obtain can be concluded as follows.

Theorem 1.1. (i) There exist a function $f \in L^{\infty}(\mathbb{R})$ and a nonzero measure set $E \subset \mathbb{R}$ such that for any $x \in E, S_{\mathcal{I}} f(x)=\infty$ for some $\mathcal{I}$, whence $S f(x)=\infty$.

(ii) There exist a compactly supported function $f \in L^{\infty}(\mathbb{R})$ and $\mathcal{I}$ such that $S_{\mathcal{I}} f \notin B M O_{d}(\mathbb{R})$.

The (ii) is interesting in the sense that it is different from [4, where the author proved that the classical $g$-function is bounded from $L_{c}^{\infty}(\mathbb{R})$ to $B M O(\mathbb{R})$ even though we can find $f \in L^{\infty}(\mathbb{R})$ such that $g(f)=\infty$ almost everywhere.

On the other hand, from (i), we cannot expect $S$ to map the whole $L^{\infty}$ to $B M O_{d}$. Moreover, by the $L^{2}$-boundedness (1.4), for almost every $x \in \mathbb{R}, S f(x)<\infty$ for all $f \in L_{c}^{\infty}(\mathbb{R})$. Hence the best result we can expect is the following, which will be shown in Section 3.

Theorem 1.2. $S$ is bounded from $L_{c}^{\infty}(\mathbb{R})$ to $B M O_{d}(\mathbb{R})$. Hence by interpolation, for all $2<p<\infty, S$ is bounded on $L^{p}(\mathbb{R})$, whence $S_{\mathcal{I}}$ is uniformly bounded on $L^{p}(\mathbb{R})$.

Here the interpolation can be explained as follows. First, by standard complex interpolation argument, we can prove $S$ is bounded from $L_{c}^{p}(\mathbb{R})$, the space of compactly supported $L^{p}(\mathbb{R})$ functions, to $L^{p}(\mathbb{R})$. Then the final result is obtained by density argument.

\section{Proof of Theorem 1.1}

This section is devoted to the proof of Theorem 1.1 Some comments on the cases $\mathbb{Z}, \mathbb{T}$ and $\mathbb{R}^{n}$ with $n \geq 2$ are also included at the end of this section.

Proof. The proof of (i). Take $f=\chi_{[0, \infty)}$ and $I_{k}(x)=\left[x-2^{k}, x\right)$ for any $k \in \mathbb{Z}$. For any $x \in[0, \infty)$, there exists $\ell>0$ such that $x \in\left[0,2^{\ell}\right)$. 
Fix an $x \in\left[0,2^{\ell}\right)$. Obviously, $\mathcal{E}_{k} f(x)=1$, for all $k \in \mathbb{Z}$. On the other hand, if $k>\ell$, then $x-2^{k}<0$. Thus

$$
M_{I_{k}(x)} f(x)=2^{-k} \int_{x-2^{k}}^{x} f=2^{-k} x \leq 2^{-1} .
$$

Therefore,

$$
\begin{aligned}
S_{\mathcal{I}} f(x) & \geq\left(\sum_{k>\ell}\left|M_{I_{k}(x)} f(x)-\mathcal{E}_{k} f\right|^{2}\right)^{1 / 2} \\
& \geq\left(\sum_{k>\ell}|1-1 / 2|^{2}\right)^{1 / 2}=\infty .
\end{aligned}
$$

The proof of (ii). The basic construction is similar to that in Remark 4.5 of [2], where the authors proved in the torus case. Let $I_{\ell}=\left[1 / 2,1 / 2+1 / 2^{\ell}\right)$. Let $P$ and $N$ denote two disjoint subsets of $[1 / 2,1)$ such that for all $\ell>2$,

$$
\left|P \cap I_{\ell}\right|=\left|N \cap I_{\ell}\right|=2^{-(\ell+1)} .
$$

Take $I_{k}(x)=\left(x-2^{k}, x\right]$ for each $x \in N$, and $I_{k}(x)=\left(x, x+2^{k}\right]$ for each $x \in P$. Let $f=\chi_{[1 / 2,1)}$ and let $\ell>2$.

Fix $x \in I_{\ell} . \mathcal{E}_{k} f(x)=1$ for $k \leq-2 ; \mathcal{E}_{k} f(x)=2^{-k-1}$ for $k \geq-1$. Moreover, if $x \in P$, then

$$
M_{I_{k}(x)} f(x)=2^{-k} \int_{x}^{x+2^{k}} f=1, \text { for } k \leq-2 ;
$$

$M_{I_{k}(x)} f(x)=2^{-k}(1-x)$ for $k \geq-1$. If $x \in N$, then for $k>-\ell, x-2^{k}<1 / 2$. Thus

$$
M_{I_{k}(x)} f(x)=2^{-k} \int_{x-2^{k}}^{x} f=2^{-k}(x-1 / 2) \leq 2^{-k-\ell}<1 / 2 .
$$

To conclude, for $x \in P \cap I_{\ell}$, we have

$$
\begin{aligned}
S_{\mathcal{I}} f(x) & \leq\left(\sum_{k \in \mathbb{Z}}\left|M_{I_{k}(x)} f(x)-\mathcal{E}_{k} f\right|^{2}\right)^{1 / 2} \\
& =\left(\sum_{k \geq-1}\left|M_{I_{k}(x)} f(x)-\mathcal{E}_{k} f\right|^{2}\right)^{1 / 2} \\
& =\left(\sum_{k \geq-1}\left|2^{-k}(x-1 / 2)\right|^{2}\right)^{1 / 2} \leq 1 .
\end{aligned}
$$

While for $x \in N \cap I_{\ell}$, we have

$$
\begin{aligned}
S_{\mathcal{I}} f(x) & \geq\left(\sum_{\ell<k \leq-2}\left|M_{I_{k}(x)} f(x)-\mathcal{E}_{k} f\right|^{2}\right)^{1 / 2} \\
& =\left(\sum_{\ell<k \leq-2}|1-1 / 2|^{2}\right)^{1 / 2}=\frac{1}{2} \sqrt{\ell-1} .
\end{aligned}
$$

Then, it is easy to check that for large $\ell$,

$$
\left|\int_{N \cap I_{\ell}}(S f(x)-S f(y)) d y\right| \geq 2\left|\int_{P \cap I_{\ell}}(S f(x)-S f(y)) d y\right|
$$


for any $x \in P \cap I_{\ell}$. Therefore, by triangle inequalities,

$$
\begin{aligned}
& \|S f\|_{B M O_{d}} \geq \frac{1}{\left|I_{\ell}\right|^{2}} \int_{I_{\ell}}\left|\int_{I_{\ell}}(S f(x)-S f(y)) d y\right| d x \\
& \geq \frac{1}{\left|I_{\ell}\right|^{2}} \int_{P \cap I_{\ell}}\left|\int_{N \cap I_{\ell}}(S f(x)-S f(y)) d y+\int_{P \cap I_{\ell}}(S f(x)-S f(y)) d y\right| d x \\
& \geq \frac{1}{\left|I_{\ell}\right|^{2}} \int_{P \cap I_{\ell}}\left(\left|\int_{N \cap I_{\ell}}(S f(x)-S f(y)) d y\right|-\left|\int_{P \cap I_{\ell}}(S f(x)-S f(y)) d y\right|\right) d x \\
& \geq \frac{1}{2} \frac{1}{\left|I_{\ell}\right|^{2}} \int_{P \cap I_{\ell}}\left|\int_{N \cap I_{\ell}}(S f(x)-S f(y)) d y\right| d x \\
& \geq \frac{1}{2} \frac{1}{\left|I_{\ell}\right|^{2}} \int_{P \cap I_{\ell}} \int_{N \cap I_{\ell}}\left(\frac{1}{2} \sqrt{\ell-1}-1\right) d y d x \geq \frac{1}{16} \sqrt{\ell} .
\end{aligned}
$$

This finishes the proof since $\ell$ can be taken as large as we want.

In the cases $\mathbb{T}, \mathbb{Z}$ and $\mathbb{R}^{n}$, we can define $S_{\mathcal{I}}\left(\right.$ or $S_{\mathcal{Q}}$ ) and $S$ similarly.

Remark 2.1. (i). The case $\mathbb{Z}$. Take $f=\chi_{[0, \infty)}$ and $I_{k}(j)=\left[j-2^{k}, j\right)$ for any $k \in \mathbb{N}$. Then using the same arguments, we can show an analog of Theorem 1.1 (i). However, Theorem 1.1 (ii) can never be true in this case. Actually it follows from Theorem A' of [3] that $S_{\mathcal{I}} f^{\prime}$ 's belong to $L^{2}(\mathbb{Z}) \subset L^{\infty}(\mathbb{Z}) \subset B M O_{d}(\mathbb{Z})$ for any $f \in L_{c}^{\infty}(\mathbb{Z}) \subset L^{2}(\mathbb{Z})$.

(ii). The case $\mathbb{T}$. Theorem 1.1 can never be true in this case since the $S_{\mathcal{I}} f$ 's belong to $L^{2}(\mathbb{T})$, whence finite almost everywhere for any $f \in L^{\infty}(\mathbb{T}) \subset L^{2}(\mathbb{T})$ by Theorem 2.2 in [2]. An analog of Theorem 1.1] (ii) has been obtained in Remark 4.5 in $[2]$.

(iii). The case $\mathbb{R}^{n}$. Let us explain in the case $n=2$ to simplify the notation. An analog of Theorem 1.1 (i) is true by taking $f=\chi_{[0, \infty) \times[0, \infty)}$ and $Q_{k}(j, \ell)=$ $\left[j-2^{k}, j\right) \times\left[\ell-2^{k}, \ell\right)$ using similar arguments. On the other hand, we can show an analog of Theorem 1.1 (ii) using similar calculations by considering $f$ and $\mathcal{Q}$ as follows. Let $f=\chi_{[1 / 2,1)}$. Let $Q_{\ell}=\left[1 / 2,1 / 2+1 / 2^{\ell}\right) \times\left[1 / 2,1 / 2+1 / 2^{\ell}\right)$. Let $P$ and $N$ denote two disjoint subsets of $[1 / 2,1) \times[1 / 2,1)$ such that for all $\ell>2$,

$$
\left|P \cap Q_{\ell}\right|=\left|N \cap Q_{\ell}\right|=2^{-2(\ell+1)} .
$$

Take $Q_{k}(x, y)=\left(x-2^{k}, x\right] \times\left(y-2^{k}, y\right]$ for each $(x, y) \in N$, and $Q_{k}(x, y)=$ $\left(x, x+2^{k}\right] \times\left(y, y+2^{k}\right]$ for each $(x, y) \in P$.

\section{Proof of Theorem 1.2}

This section is devoted to the proof of Theorem 1.2. Moreover, we can verify that the following argument works also in the case $\mathbb{Z}, \mathbb{T}$ and $\mathbb{R}^{n}$ with $n \geq 2$. We leave the details to the interested reader.

Proof. It suffices to prove that there exists a positive constant $C$ such that

$$
\|S f\|_{B M O_{d}} \leq C\|f\|_{\infty}, \forall f \in L_{c}^{\infty}(\mathbb{R}) .
$$

We shall use the equivalent definition of $B M O_{d}$ norm,

$$
\|g\|_{B M O_{d}} \simeq \sup _{I \text { dyadic }} \inf _{a_{I}} \frac{1}{|I|} \int_{I}\left|g-a_{I}\right| .
$$

Give $f \in L^{\infty}(\mathbb{R})$, and a dyadic interval $I$. We decompose $f$ as $f=f \mathbb{1}_{I^{*}}+$ $f \mathbb{1}_{\mathbb{R} \backslash I^{*}}=f_{1}+f_{2}$, where $I^{*}$ is the cube with the same center as $I$ but three times 
the side-length. We shall take $a_{I}=S f_{2}\left(c_{I}\right)$ where $c_{I}$ is the center of $I$. Write $S f-a_{I}$ as

$$
S f-a_{I}=S f-S f_{2}+S f_{2}-a_{I},
$$

by triangle inequalities,

$$
\begin{aligned}
& \frac{1}{|I|} \int_{I}\left|S f-a_{I}\right| \leq \frac{1}{|I|} \int_{I}\left|S f-S f_{2}\right|+\frac{1}{|I|} \int_{I}\left|S f_{2}(x)-S f_{2}\left(c_{I}\right)\right| d x \\
& \leq \frac{1}{|I|} \int_{I}\left(\sum_{k} \sup _{J \in \mathcal{I}_{k}}\left|M_{J+x} f_{2}(x)-\mathcal{E}_{k} f_{2}(x)-\left(M_{J+x} f_{2}\left(c_{I}\right)-\mathcal{E}_{k} f_{2}\left(c_{I}\right)\right)\right|^{2}\right)^{\frac{1}{2}} \\
& +\frac{1}{|I|} \int_{I}\left|S f_{1}\right|=(1)+(2) .
\end{aligned}
$$

The first term (1) is easily estimated by the fact that $S$ is of strong type $(2,2)$. Indeed,

$$
(1) \leq\left(\frac{1}{|I|} \int_{I}\left|S\left(f_{1}\right)\right|^{2}\right)^{\frac{1}{2}} \leq C\left(\frac{1}{|I|} \int_{I}\left|f_{1}\right|^{2}\right)^{\frac{1}{2}} \leq C\|f\|_{\infty}
$$

The second term (2) is controlled by a constant multiple of $\|f\|_{\infty}$ once we prove that for any $x \in I$ and any $J \in \mathcal{I}_{k}$,

$$
\left(\sum_{k}\left|M_{J+x} f_{2}(x)-\mathcal{E}_{k} f_{2}(x)-\left(M_{J+x} f_{2}\left(c_{I}\right)-\mathcal{E}_{k} f_{2}\left(c_{I}\right)\right)\right|^{2}\right)^{\frac{1}{2}} \leq C\|f\|_{\infty} .
$$

If $2^{k}<|I|$, then $\mathcal{E}_{k} f_{2}$ is supported in $I$ and $J+x$ is contained in $I^{*}$ since

$$
\left|x+y-c_{I}\right| \leq\left|x-c_{I}\right|+|y| \leq 1 / 2|I|+2^{k+1} \leq 3|I|
$$

for any $y \in J$. Then, in this case, we get

$$
M_{J+x} f_{2}(x)-\mathcal{E}_{k} f_{2}(x)-\left(M_{J+x} f_{2}\left(c_{I}\right)-\mathcal{E}_{k} f_{2}\left(c_{I}\right)\right)=0, \text { for any } x \in I .
$$

Hence it suffices to consider the case $2^{k} \geq|I|$. Note that, in this case, $I$ should be contained in some atom of $\sigma_{k}$, so $\mathcal{E}_{k} f_{2}(x)=\mathcal{E}_{k} f_{2}\left(c_{I}\right)$. On the other hand,

$$
\begin{aligned}
\mid M_{J+x} f_{2}(x)- & M_{J+x} f_{2}\left(c_{I}\right)\left|=2^{-k}\right| \int_{J+x} f_{2}-\int_{J+c_{I}} f_{2} \mid \\
& =2^{-k}\left|\int_{J+x \backslash J+c_{I}} f_{2}-\int_{J+c_{I} \backslash J+x} f_{2}\right| \\
& \leq 2^{-k} \int_{J+x \backslash J+c_{I}}\left|f_{2}\right|+2^{-k} \int_{J+c_{I} \backslash J+x}\left|f_{2}\right| \\
& \leq 2^{-k}\left|(J+x) \triangle\left(J+c_{I}\right)\right|\|f\|_{\infty} \leq C 2^{-k}|I|\|f\|_{\infty} .
\end{aligned}
$$

The last inequality is due to the fact that $\left|(J+x) \triangle\left(J+c_{I}\right)\right| \leq C\left|x-c_{I}\right| \leq C|I|$. Finally, the fact that $\ell^{2}$ norm is not larger than $\ell^{1}$ norm implies

$$
(2) \leq C|I|\|f\|_{\infty} \sum_{2^{k} \geq|I|} \frac{1}{2^{k}} \leq C\|f\|_{\infty} .
$$




\section{REFERENCES}

[1] James T. Campbell, Roger L. Jones, Karin Reinhold, and Máté Wierdl, Oscillation and variation for the Hilbert transform, Duke Math. J. 105 (2000), no. 1, 59-83, DOI 10.1215/S00127094-00-10513-3. MR1788042(2001h:42021)

[2] Roger L. Jones, Robert Kaufman, Joseph M. Rosenblatt, and Máté Wierdl, Oscillation in ergodic theory, Ergodic Theory Dynam. Systems 18 (1998), no. 4, 889-935, DOI 10.1017/S0143385798108349. MR 1645330(2000b:28019)

[3] Roger L. Jones, Joseph M. Rosenblatt, and Máté Wierdl, Oscillation in ergodic theory: higher dimensional results, Israel J. Math. 135 (2003), 1-27, DOI 10.1007/BF02776048. MR.1996394 (2004h:37005)

[4] Si Lei Wang, Some properties of Littlewood-Paley's g-function, Sci. Sinica Ser. A 28 (1985), no. 3, 252-262. MR794651 (87h:42031)

Instituto de Ciencias Matemáticas, CSiC-UAM-UC3M-UCM, Consejo Superior de Investigaciones Científicas, C/Nicolás Cabrera 13-15, 28049, Madrid, Spain

E-mail address: guixiang.hong@icmat.es 